\title{
Is synchronised NIPPV more effective than NIPPV and NCPAP in treating apnoea of prematurity (AOP)? A randomised cross-over trial
}

\author{
Camilla Gizzi, ${ }^{1}$ Francesco Montecchia, ${ }^{2}$ Valentina Panetta, ${ }^{3}$ Chiara Castellano, ${ }^{1}$ \\ Chiara Mariani, ${ }^{1}$ Maristella Campelli, ${ }^{1}$ Paola Papoff, ${ }^{4}$ Corrado Moretti, ${ }^{4}$ \\ Rocco Agostino ${ }^{1}$
}

${ }^{1}$ Neonatal Intensive Care Unit, Pediatric and Neonatal Department, "S.Giovanni Calibita" Fatebenefratelli Hospital, Rome, Italy ${ }^{2}$ Medical Engineering Laboratory, Department of Civil Engineering and Computer Science Engineering, "Tor Vergata" University of Rome, Rome, Italy

${ }^{3}$ SeSMIT-A.Fa.R., Medical Statistics \& Information Technology, Fatebenefratelli Association for Biomedical and Sanitary Research, Rome, Italy ${ }^{4}$ Pediatric Emergency and Intensive Care, Department of Pediatrics, Policlinico "Umberto I," Sapienza University of Rome, Rome, Italy

\section{Correspondence to} Dr Camilla Gizzi, Neonatal Intensive Care Unit, Pediatric and Neonatal Department,

"S.Giovanni Calibita" Fatebenefratelli Hospital,

Piazza Confienza,

3 Rome 00185, Italy;

camillagizzi@tin.it

Received 25 December 2013 Revised 22 September 2014 Accepted 23 September 2014 Published Online First 15 October 2014

\section{SLinked}

- http://dx.doi.org/10.1136/

fetalneonatal-2013-305830

- http://dx.doi.org/10.1136/

fetalneonatal-2014-306109

CrossMark

To cite: Gizzi $C$

Montecchia F, Panetta $V_{\text {, }}$

et al. Arch Dis Child Fetal

Neonatal Ed 2015;100:

F17-F23.

\section{ABSTRACT}

Background Apnoea, desaturations and bradycardias are common problems in preterm infants which can be treated with nasal continuous positive airway pressure (NCPAP) and nasal intermittent positive pressure ventilation (NIPPV). It is unclear whether synchronised NIPPV (SNIPPV) would be even more effective.

Objective To assess the effects of flow-SNIPPV, NIPPV and NCPAP on the rate of desaturations and bradycardias in preterm infants and, secondarily, to evaluate their influence on pattern of breathing and gas exchange.

Patients and methods Nineteen infants (mean gestational age at study 30 weeks, 9 boys) with apnoeic spells were enrolled in a randomised controlled trial with a cross-over design. They received flow-SNIPPV, NIPPV and NCPAP for $4 \mathrm{~h}$ each. All modes were provided by a nasal conventional ventilator able to provide synchronisation by a pneumotachograph. The primary outcome was the event rate of desaturations $(\leq 80 \%$ arterial oxygen saturation) and bradycardias ( $\leq 80 \mathrm{bpm})$ per hour, obtained from cardiorespiratory recordings. The incidence of central apnoeas ( $\geq 10 \mathrm{~s}$ ) as well as baseline heart rate, $\mathrm{FiO}_{2}, \mathrm{SpO}_{2}$, transcutaneous blood gases and respiratory rate were also evaluated.

Results The median event rate per hour during flowSNIPPV, NIPPV and NCPAP was 2.9, 6.1 and 5.9, respectively ( $p<0.001$ and 0.009 , compared with flowSNIPPV). Central apnoeas per hour were 2.4, 6.3 and 5.4 , respectively ( $p=0.001$, for both compared with flow-SNIPPV), while no differences in any other parameter studied were recorded.

Conclusions Flow-SNIPPV seems more effective than NIPPV and NCPAP in reducing the incidence of desaturations, bradycardias and central apnoea episodes in preterm infants.

\section{INTRODUCTION}

Apnoea of prematurity (AOP) is a common problem in preterm infants. Desaturations and bradycardias, considered as mainly elicited by AOP, are associated with longer-term neurodevelopmental problems. ${ }^{1-4}$ Standard therapies to treat these conditions are nasal continuous positive airway pressure (NCPAP) and methylxanthines. For infants who do not respond to this combined strategy, nasal intermittent positive pressure ventilation (NIPPV) has been proposed as an alternative to $\mathrm{NCPAP}^{5-7}$ as it may provide a greater respiratory

\section{What is already known on this topic}

Apnoea, desaturations and bradycardias are common problems in preterm infants related to longer-term adverse outcome.

- Nasal continuous positive airway pressure or nasal intermittent positive pressure ventilation (NIPPV) has been shown to improve these symptoms.

- It is unclear whether synchronised NIPPV works even better.

\section{What this study adds}

- Synchronised nasal intermittent positive pressure ventilation (NIPPV) significantly improved the overall incidence of desaturations and bradycardias.

- Synchronised NIPPV effectively reduced central apnoeas compared with NIPPV and nasal continuous positive airway pressure.

support. It is unclear whether synchronised NIPPV (SNIPPV) would be even more effective, as synchronisation may add some favourable effects. Our aim was thus to compare the effects of flow-SNIPPV, NIPPV and NCPAP on the rate of desaturation events and bradycardias in preterm infants and, secondarily, to evaluate their influence on pattern of breathing and gas exchange. Our hypothesis was that flow-SNIPPV is more effective than NIPPV and NCPAP.

\section{METHODS}

\section{Patients}

Between October 2010 and February 2012, newborn infants admitted to the neonatal intensive care unit (NICU) at "S. Giovanni Calibita" Fatebenefratelli Hospital of Rome were screened for eligibility. Inclusion criteria were: (1) gestational age $<34$ weeks by maternal dates or early ultrasound, (2) postmenstrual age at time of study $\leq 38$ weeks, (3) requirement for NCPAP to treat AOP and (4) fraction of inspired oxygen $\left(\mathrm{FiO}_{2}\right)$ need $<0.3$. At the time of study, all infants were receiving caffeine citrate $(5 \mathrm{mg} / \mathrm{kg} /$ day $)$ and were in stable conditions except for their apnoeic episodes. 
Infants with congenital or chromosomal abnormalities, acute infections, anaemia, electrolyte imbalances, intraventricular haemorrhage or patent ductus arteriosus were excluded. Written informed parental consent was obtained for each infant.

\section{Study design and protocol}

A randomised cross-over study with three treatment phases was conducted. Following recruitment, infants were randomly allocated to the six possible sequences of flow-SNIPPV, NIPPV and NCPAP applied for $4 \mathrm{~h}$ each. Four patients were allocated to sequence NIPPV/NCPAP/SNIPPV, while three patients were allocated to each of the remaining five sequences.

The sequence to be followed in each patient was revealed to the investigators immediately before recording by a telephone call. Throughout the study, infants were fed at $3 \mathrm{~h}$ intervals and received their routine care while in an incubator at thermoneutrality and in a supine position. The study protocol was approved by the Ethics Committee of the hospital.

\section{Respiratory support system}

Flow-SNIPPV, NIPPV and NCPAP were applied using 'Giulia' neonatal ventilator via nasal prongs (Ginevri Medical Technologies, Rome, Italy). Synchronisation was obtained by means of a fixed orifice pneumotachograph $(2.5 \mathrm{~mm}$ inner diameter-dead space $1 \mathrm{~mL}$ ) interposed between the prongs and the $\mathrm{Y}$ piece. The largest possible prongs were used, with a snug fit to avoid leakage. No precautions were taken to avoid leakage from the mouth. For all ventilation modes initial flow was set at $8 \mathrm{~L} / \mathrm{min}$ and $\mathrm{FiO}_{2}$ adjusted to keep pulse oximeter saturation $\left(\mathrm{SpO}_{2}\right)$ between $90 \%$ and $94 \%$. The system was regularly monitored and flow delivered by the ventilator adjusted in order to keep positive end-expiratory pressure constant on selected values. Flow-SNIPPV was delivered in the assist/control mode (ie, the ventilator assisted each spontaneous breath) with the following parameters: Ti $0.3-0.35 \mathrm{~s}$, peak inspiratory pressure (PIP) $15 \mathrm{~cm} \mathrm{H}_{2} \mathrm{O}$, positive end-expiratory pressure $5-6 \mathrm{~cm} \mathrm{H}{ }_{2} \mathrm{O}$ and back-up rate $20 \mathrm{bpm}$. Trigger level was initially set at $0.1 \mathrm{~L} / \mathrm{min}$, then adjusted for each infant in order to obtain the best synchronisation and avoid autotriggering. Synchronisation was continuously monitored during SNIPPV by one of the authors who attended the registration, looking at the ventilator's and the infant's spontaneous respiratory rate (RR). In order to facilitate the detection of synchrony, synchronous breaths are displayed on the ventilator's screen as a bicolour pressure trace, green for inspiration and white for expiration, while mandatory mechanical breaths are displayed as a white pressure trace during inspiration and expiration. To avoid autotriggering and low signal detection, the nose of the infants, the flow sensor and the ventilator circuit were regularly inspected and kept clear of secretion and humidity. NIPPV was delivered using the same parameters as flow-SNIPPV at a mechanical RR of $20 \mathrm{bpm}$. During flow-SNIPPV and NIPPV, peak pressure could be increased up to $\max 20 \mathrm{~cm} \mathrm{H}_{2} \mathrm{O}$ to obtain effective aeration per auscultation and according to blood gases. NCPAP was set at $5-6 \mathrm{~cm} \mathrm{H}_{2} \mathrm{O}$.

\section{Cardiorespiratory recordings}

Throughout the study the following signals were monitored and recorded (Infinity Delta XL Monitor and GateWay Plus computerised polygraphic system, Dräger Medical System, Lübeck, Germany): thoracic impedance and RR, pulse oximetry waveform and $\mathrm{SpO}_{2}$, ECG waveform and beat-to-beat heart rate (HR), transcutaneous $\mathrm{O}_{2}$ and $\mathrm{CO}_{2}$ pressures (PtcO2 and $\mathrm{PtcCO}_{2}$ ). RR, $\mathrm{SpO}_{2}, \mathrm{HR}$ and transcutaneous blood gases were in $2 \mathrm{~s}$ averaging mode. Airway pressure, airway flow and $\mathrm{FiO}_{2}$ from 'Giulia' Ventilator were also recorded. Sensors applied to infants and their output signals were regularly checked during recordings. Physiological signals were simultaneously recorded on a computer (Compaq 6720s, Hewlett-Packard, Palo Alto, California, USA) and afterwards imported on specific software developed on the MATLAB platform, to be analysed off-line. To improve the detection of central apnoeas, the thoracic impedance signal was low-pass filtered to remove the contribution of the cardiac signal that might be mistaken for breathing. ${ }^{8}$ The cut-off frequency of low-pass filter was obtained by fast Fourier transform of the thoracic impedance signal and set according to the highest RR appearing during the whole monitored time for each infant. Recordings were analysed without access to clinical data. A desaturation was defined as a fall in $\mathrm{SpO}_{2}$ to $\leq 80 \%$ for $>1 \mathrm{~s}$. and a bradycardia as a fall in HR to $\leq 80 \mathrm{bpm}$ for more than one beat. Desaturations and bradycardias associated with a distorted signal immediately prior to their onset were excluded. A central apnoea was scored if the filtered thoracic impedance signal showed an absence of spontaneous chest wall movement due to breathing for $\geq 10 \mathrm{~s}$. Simultaneous thoracic impedance and ECG signals were analysed on the occasion of each bradycardia to exclude cardiac artefacts on apnoea detection. Desaturations and bradycardias were considered as temporally related to an apnoeic spell if their onset occurred within $15 \mathrm{~s}$ from the onset of a central apnoea. Mixed and obstructive apnoeas were not analysed. Baseline RR, HR, $\mathrm{SpO}_{2}, \mathrm{tcPO}_{2}$ and tcPCO 2 were defined as the mean of the respective parameter within artefact-free recording time and calculated using a MATLAB based software. Finally, event rates for desaturations, bradycardias and central apnoeas were calculated as the number of respective events per hour of artefact-free recording time.

\section{Statistical methods}

Sample size calculation was based on a preliminary analysis including six patients treated with NCPAP that showed a mean (SD) of 5.5 (1.8) desaturation and/or bradycardia events per hour, considered as our primary outcome. As NIPPV seems to offer no advantages over NCPAP to treat apnoea, ${ }^{5} 9$ we hypothesised that flow-SNIPPV could reduce these adverse events by $40 \%$. Assuming a common SD for all modes of ventilation, 19 participants were considered sufficient to detect a significant reduction of $40 \%$ (from 5.5 to 3.6 desaturation and/or bradycardia events per hour) using flow-SNIPPV compared with NIPPV and NCPAP, considering 0.05 type I and 0.2 type II errors and taking into account multiple comparisons.

Descriptive statistics as numbers and percentages as well as median and IQR were used to summarise demographic and clinical characteristics of the enrolled infants. Sequence, carryover

\begin{tabular}{|c|c|}
\hline Birth weight (g) & $800(678-1068)$ \\
\hline Gestational age at birth (weeks) & $27(25-28)$ \\
\hline Boys & $9(47)$ \\
\hline Body weight* $(\mathrm{g})$ & $986(722-1168)$ \\
\hline Postmenstrual age* (weeks) & $30(29-31)$ \\
\hline $\mathrm{FiO}_{2}{ }^{*}$ & $0.21(0.21-0.26)$ \\
\hline Haematocrit* $(\%)$ & $36(32-40)$ \\
\hline Days on caffeine* & $20(13-29)$ \\
\hline Milk intake* $(\mathrm{mL} / \mathrm{kg})$ & $96(68-137)$ \\
\hline
\end{tabular}


Table 2 Desaturation $(\leq 80 \%)$ and bradycardia $(\leq 80 \mathrm{bpm})$ events per hour of artefact-free recording time $(n=19)$

\begin{tabular}{|c|c|c|c|c|c|c|}
\hline & \multicolumn{3}{|c|}{ Mode of ventilation } & \multirow[b]{2}{*}{ p Value* } & \multicolumn{2}{|c|}{ SNIPPV $(p) \dagger$} \\
\hline & SNIPPV & NIPPV & NCPAP & & vs NIPPV & vs NCPAP \\
\hline Desaturations and/or bradycardias & $2.9(0.75-6.8)$ & $6.1(3.1-9.4)$ & $5.9(2-10.3)$ & $<0.001$ & 0.009 & $<0.001$ \\
\hline Desaturations $\leq 80 \%$ & $2.9(0.5-7.2)$ & $6.1(2.9-9.4)$ & $5(1.2-10.1)$ & $<0.001$ & 0.059 & 0.046 \\
\hline Bradycardias $\leq 80 \mathrm{bpm}$ & $0(0-0.48)$ & $0(0-0.52)$ & $0.24(0-1.4)$ & 0.025 & 1.000 & 0.195 \\
\hline
\end{tabular}

and period effects were analysed using a linear mixed model. Due to the small sample size and the repeated measures in the same patient, a non-parametric Friedman's test was used for comparisons between treatment modes. A post hoc analysis was done using Bonferroni adjustment if a global test revealed significant differences. A $p$ value $<0.05$ was considered statistically significant. All analyses were carried out with STATA V.12.1.

\section{RESULTS}

Within the study period, 24 infants met the inclusion criteria, but only 19 parents gave consent. Demographic and clinical characteristics of enrolled infants are presented in table 1. Sixteen $(84.2 \%)$ of them were treated with invasive mechanical ventilation for respiratory distress. In these infants intubation lasted mean (SD) 9.4 (11.3) days and they were studied at mean (SD) 12.8 (5.7) days after extubation. All infants received surfactant, three of them by means of the INSURE approach. All patients were on NCPAP at the time of study. Nine $(47.4 \%)$ infants were still on oxygen at enrolment.

As regards the primary study outcome, the median rate of desaturation events and/or bradycardias per hour during flow-SNIPPV was significantly lower than during NIPPV (2.9 vs 6.1, p=0.009) and NCPAP (2.9 vs 5.9, p<0.001) (table 2). No significant effect was found for sequence $(p=0.499)$, carryover $(p=0.451)$ and period $(p=0.271)$. After adjusting, mean differences from SNIPPV were 3.10 (95\% CI 1.5 to 4.7) for NIPPV and 2.74 (95\% CI 1.2 to 4.3 ) for NCPAP.

The fraction of desaturations occurring in association with central apnoeas were mean (SD) 0.46 (0.36) during SNIPPV, 0.55 (0.34) during NIPPV and $0.51(0.33)$ during NCPAP, $(p=0.183)$. Four (21\%) infants showed no bradycardias during the whole registration period. Of all the recorded bradycardias, mean (SD) 82.3 (15.6)\% were related to an apnoeic spell.

Regarding the secondary study variables (table 3 ), a decrease in central apnoeas per hour was observed during flow-SNIPPV compared with NIPPV (2.4 vs 5.3, p=0.001) and NCPAP (2.4 vs 6.3, $\mathrm{p}=0.001)$. No significant effect was found for sequence $(p=0.529)$, carryover $(p=0.111)$ and period $(p=0.806)$. Baseline cadiorespiratory parameters were significantly different during the three modes of non-invasive ventilation (NIV) according to the Friedman test, but these differences were lost after Bonferroni adjustment. The duration of the hypoxic events and bradycardias was not influenced by the mode of ventilation. No severe adverse events were reported during the study. Figures 1-5 illustrate typical traces of airway pressure, flow and thoracic impedance recorded throughout the study. In most infants, flow-SNIPPV and NIPPV backup pressure peaks produced no chest inflations during apnoea.

\section{DISCUSSION}

While NCPAP is considered effective in treating AOP, studies on NIPPV reported conflicting results. ${ }^{5-7}$ Recently Pantalitschka, observed that variable-flow NCPAP and NIPPV were more effective than conventional ventilator NIPPV in improving symptoms related to AOP, and encouraged further studies to investigate how SNIPPV performs. According to our results, flow-SNIPPV therapy was associated with an overall reduction in the combined incidence of adverse events, desaturations and bradycardias, by about $40 \%$ when compared with NIPPV and NCPAP, and also resulted in a $50 \%$ decrease in the incidence of central apnoeas. The fraction of desaturations occurring in association with central apnoeas was similar during all modes of ventilation. No statistically significant differences were observed in the measured cardiorespiratory parameters on post hoc testing of paired groups.

Table 3 Secondary study variables $(n=19)$

\begin{tabular}{|c|c|c|c|c|c|c|}
\hline & \multicolumn{3}{|c|}{ Mode of ventilation } & \multirow[b]{2}{*}{ p Value* } & \multicolumn{2}{|c|}{ SNIPPV $(p) \dagger$} \\
\hline & SNIPPV & NIPPV & NCPAP & & vs NIPPV & vs NCPAP \\
\hline Central apnoeas ( $\geq 10 \mathrm{~s}$ ) per hour & $2.4(1-3.6)$ & $6.3(2.8-17)$ & $5.4(3.1-12)$ & 0.002 & 0.001 & 0.001 \\
\hline HR (bpm) & $156(150-161)$ & $158(149-162)$ & $159(154-163)$ & $<0.001$ & 0.481 & 0.111 \\
\hline Baseline RR (bpm) & $57(50-64)$ & $54(49-60)$ & $53(47-57)$ & 0.058 & 0.440 & 0.663 \\
\hline $\mathrm{FiO}_{2}$ & $0.22(0.21-0.25)$ & $0.21(0.21-0.26)$ & $0.22(0.21-0.26)$ & 0.000 & 0.607 & 0.814 \\
\hline $\mathrm{SpO}_{2}(\%)$ & $91(90-96)$ & $91(90-93)$ & $91(90-97)$ & 0.003 & 0.061 & 0.185 \\
\hline $\mathrm{PtcO}_{2}(\mathrm{~mm} \mathrm{Hg})$ & $58(45-71)$ & $54(49-75)$ & $53(48-68)$ & 0.020 & 1.887 & 1.015 \\
\hline $\mathrm{PtcCO}_{2}(\mathrm{~mm} \mathrm{Hg})$ & $49(46-52)$ & $52(43-58)$ & $49(42-55)$ & 0.018 & 1.849 & 0.787 \\
\hline
\end{tabular}

Data are expressed as median (IQR).

*Friedman's test.

†After Bonferroni adjustment.

HR, heart rate; NCPAP, nasal continuous positive airway pressure; NIPPV, nasal intermittent positive pressure ventilation; RR, respiratory rate; SNIPPV, synchronised NIPPV. 
Figure 1 Time tracing of delivered pressure, air flow and thoracic impedance (unfiltered (light gray) and filtered (dark gray)) showing the typical pattern recorded during an apnoeic episode while on flow-synchronised nasal intermittent positive pressure ventilation. In all traces inspiration is up. Mechanical breaths follow infant's spontaneous ones before and after the respiratory pause. No chest inflation is detected during the apnoeic spell despite mechanical backup breaths.

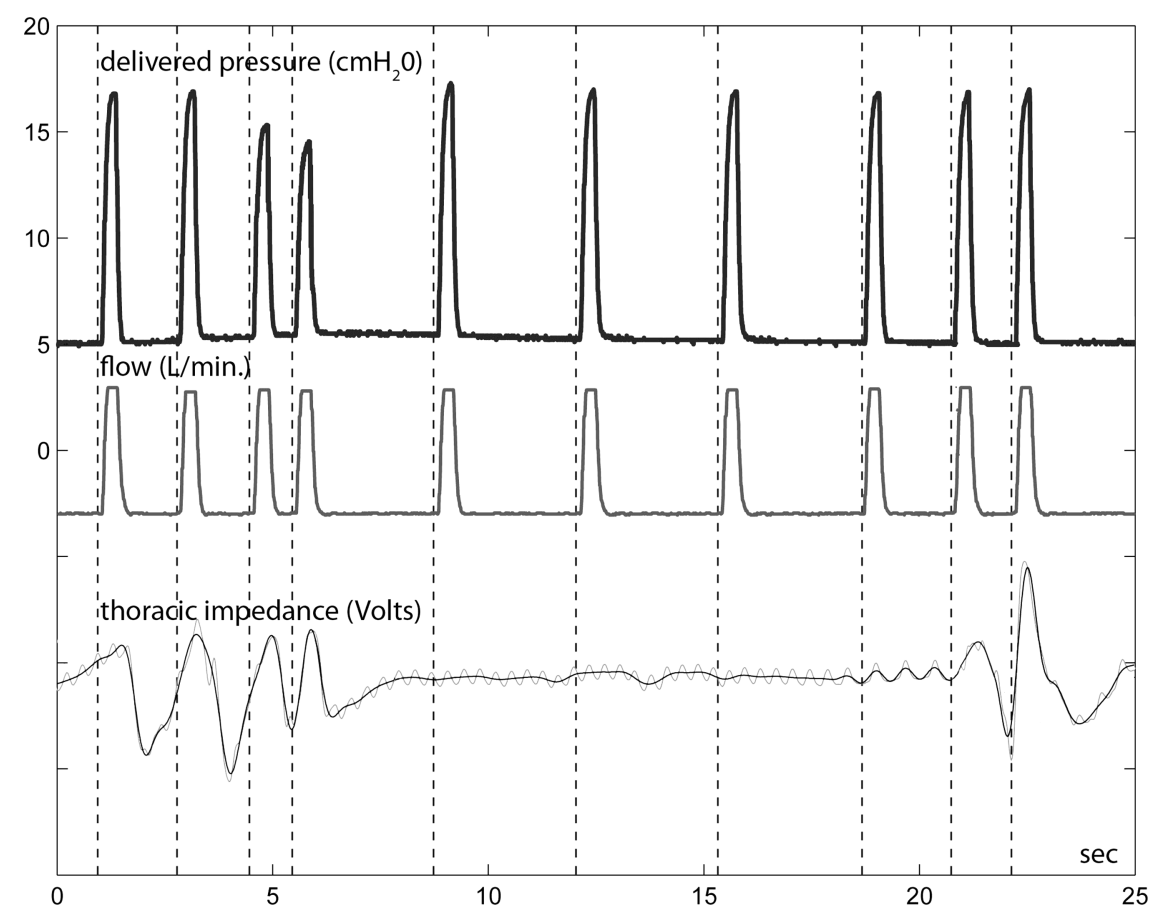

Although promising, these results should be interpreted with due caution. First, only a small number of infants was included, even though the sample size required to show statistically significant differences in the outcome data consisted of 19 infants. Furthermore, extremely low birthweight infants, that are particularly prone to adverse events, were highly represented in our population as 12 infants weighed $<1000 \mathrm{~g}$ at birth, and 9 at the time of recording. Second, the study cross-over periods were $4 \mathrm{~h}$ long, and no information on longer-term outcome can be provided.

According to our results, the efficacy of flow-SNIPPV was mostly due to a decrease in desaturation events, as the rate of bradycardias per hour was quite low among enrolled infants. We speculate that flow-SNIPPV limited oxygenation instability either by reducing the incidence of adverse events related to AOP as well as through different effects on ventilation. Preterm infants are particularly prone to loss of functional residual capacity (FRC) due to the excessive chest wall compliance, while the softness of the upper airway tissues may predispose to obstruction. Several studies showed that increasing NCPAP levels reduce the frequency of central apnoeas and periodic breathing. ${ }^{10-12}$ Nevertheless, high pressures in infants with minimal lung disease may decrease cardiac output, ${ }^{13-15}$ and increase the risk of pneumothorax. ${ }^{16}$ Alternatively, mean airway pressure (MAP) can be increased by increasing mechanical RR during NIPPV or by using SNIPPV. The latter, however, seems to be a more logical solution as synchronous
Figure 2 Time tracing of delivered pressure, air flow and thoracic impedance (unfiltered (light gray) and filtered (dark gray)) showing the typical pattern recorded during an apnoeic episode while on nasal intermittent positive pressure ventilation. In all traces inspiration is up. During the apnoeic spell, backup mechanical breaths did not result in any chest inflation.

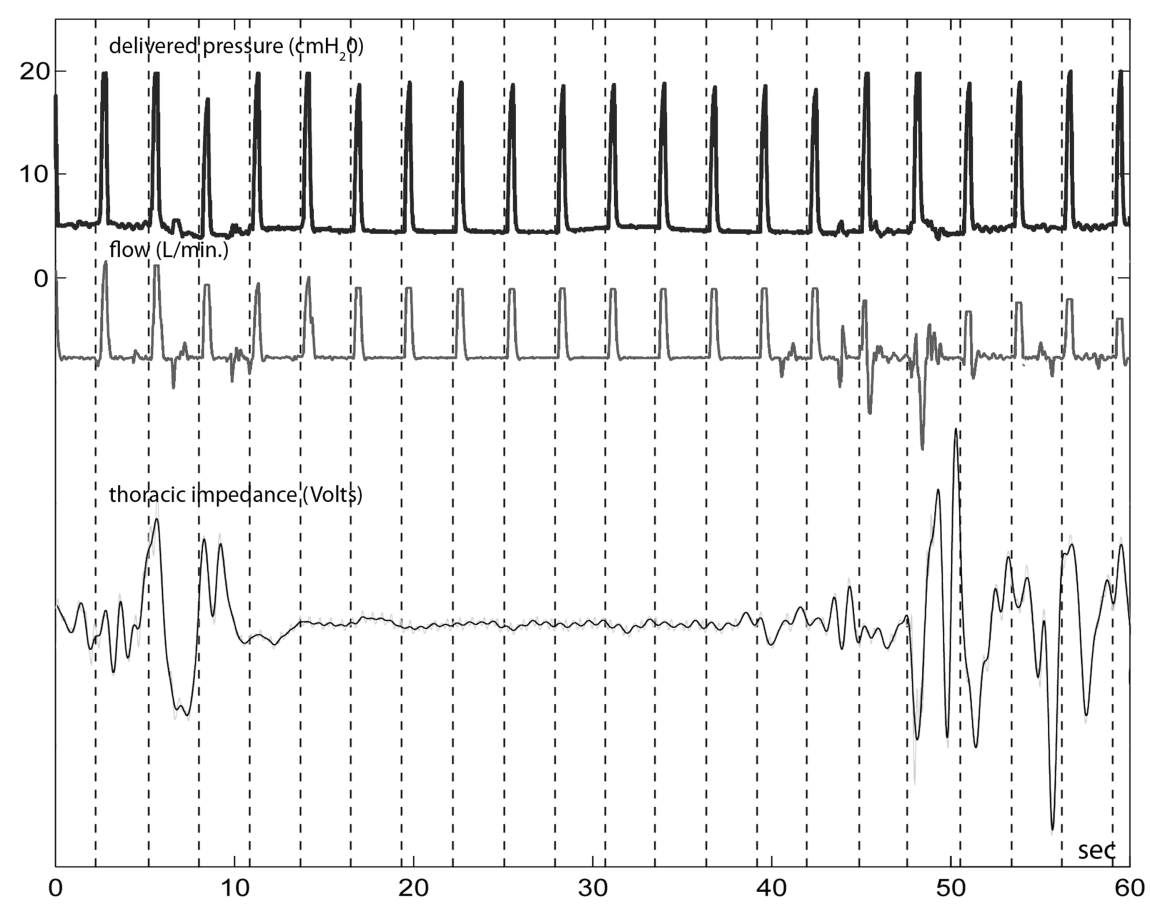

Gizzi C, et al. Arch Dis Child Fetal Neonatal Ed 2015;100:F17-F23. doi:10.1136/archdischild-2013-305892 
Figure 3 Time tracing of delivered pressure, air flow and thoracic impedance (unfiltered (light gray) and filtered (dark gray)) showing the typical pattern recorded while on flow-synchronised nasal intermittent positive pressure ventilation. In all traces inspiration is up. Mechanical breaths follow infant's spontaneous ones.

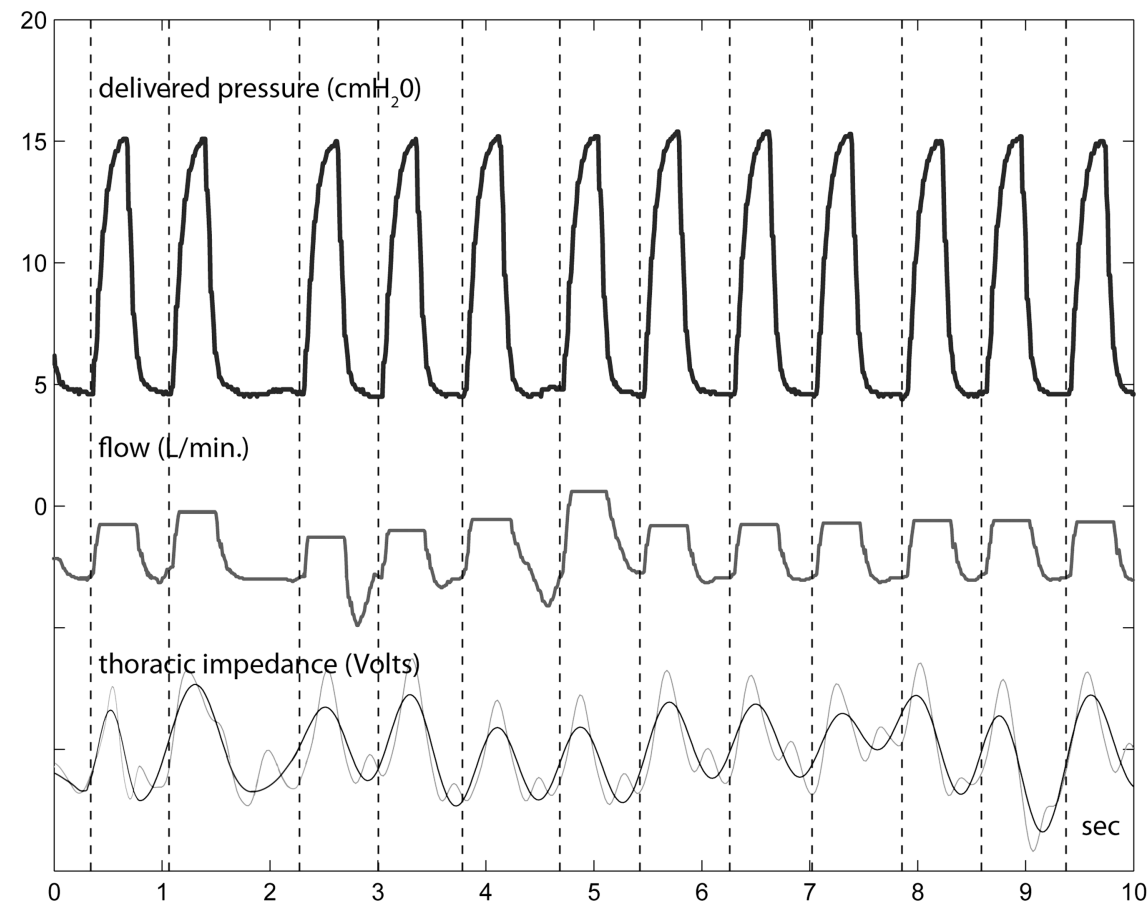

mechanical breaths, delivered when the glottis is open, may be more effectively transmitted to the lungs, improving ventilation. ${ }^{17}{ }^{18}$ Conversely, asynchronous breaths may induce laryngeal closure and inhibit inspiration, ${ }^{19} 20$ increase abdominal distention, ${ }^{21}$ cause volutrauma and pneumothorax, ${ }^{22}$ have detrimental effects on blood pressure and cerebral blood flow, ${ }^{23}$ and increase work of breathing (WOB). ${ }^{21}{ }^{24}$ Contrariwise, a reduced WOB improves hypoxaemic episodes and apnoea, ${ }^{25}$ and SNIPPV decreases WOB compared with NCPAP and NIPPV. ${ }^{17}$ 26-28

Technical issues also determine the effectiveness of synchronisation and may be responsible for our findings. Until present, two devices have been commonly used to trigger the ventilator during NIV in preterm infants: the abdominal capsule, ${ }^{29-32}$ and the flow sensor. ${ }^{17} 3334$ Recently, a device using a diaphragmatic electromyogram has been introduced, however little data are available on clinical outcomes ${ }^{35}$ The abdominal capsule, although quite effective, has some disadvantages relating to the high sensitivity to the infant's spontaneous movements and to the considerable skill required for correct placement. Studies reporting on abdominal capsule-SNIPPV for AOP as secondary outcome showed contrasting findings. ${ }^{29} 30$ Conversely, airway flow detection is a recognised and easy means for synchronising. Moreover, it offers an advantage when preterm infants employ breathing strategies with inversion of the usual sequence, that is, diaphragmatic contraction commences in advance of glottic opening, occurring in up to $60 \%$ of spontaneous breaths. ${ }^{36}$
Figure 4 Time tracing of delivered pressure, air flow and thoracic impedance (unfiltered (light gray) and filtered (dark gray)) showing the typical pattern recorded while on nasal intermittent positive pressure ventilation (NIPPV). In all traces inspiration is up. NIPPV breaths occur at different stages of the spontaneous respiratory cycle ((1) peak of breath,

(2) mid-expiration, (3) late expiration,

(4) peak of breath, (5) early expiration,

(6) mid-expiration). Infant seems not to be entrained with the ventilator.

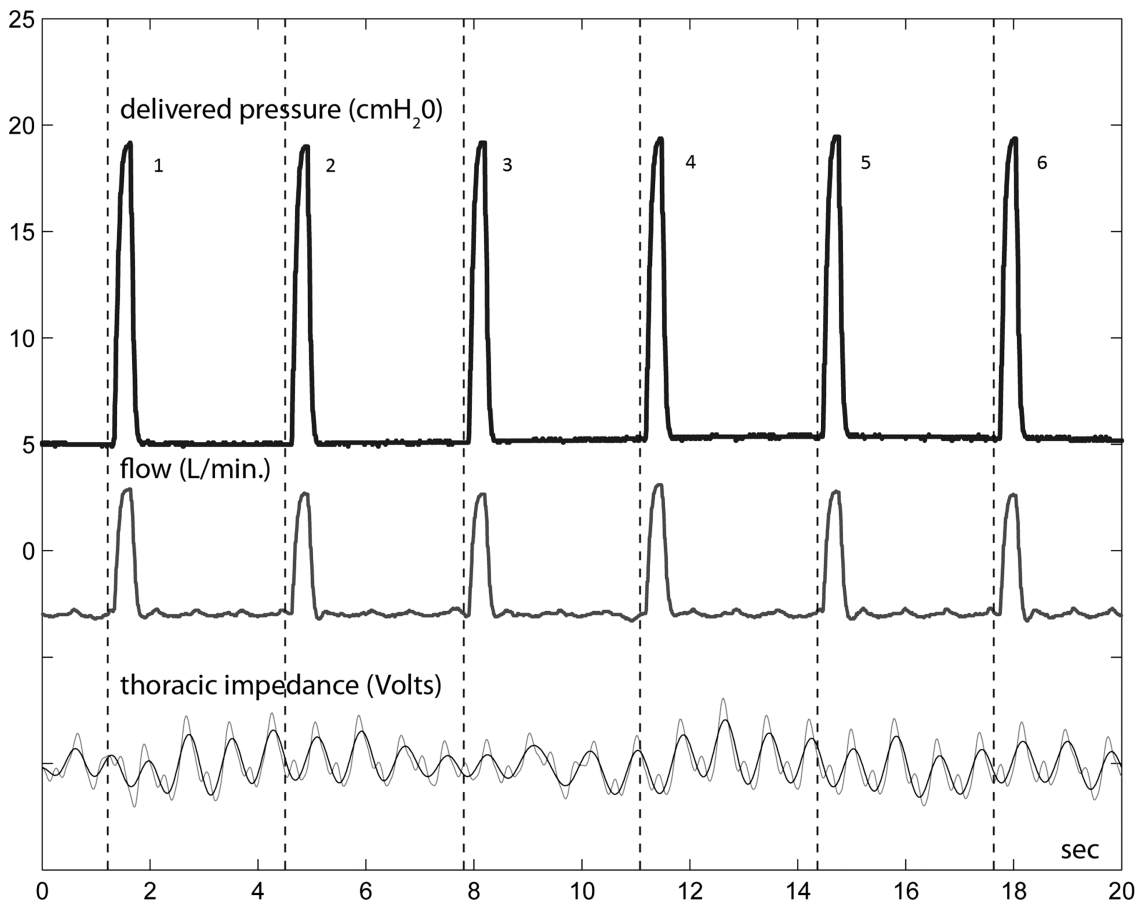

Gizzi C, et al. Arch Dis Child Fetal Neonatal Ed 2015;100:F17-F23. doi:10.1136/archdischild-2013-305892 
Figure 5 Time tracing of delivered pressure, air flow and thoracic impedance (unfiltered (light gray) and filtered (dark gray)) showing the typical pattern recorded while on nasal intermittent positive pressure ventilation (NIPPV). In all traces inspiration is up. During this recording most of NIPPV breaths occur at early inspiration, while the second breath occurred at mid-expiration, altering the infant's respiratory rhythm.

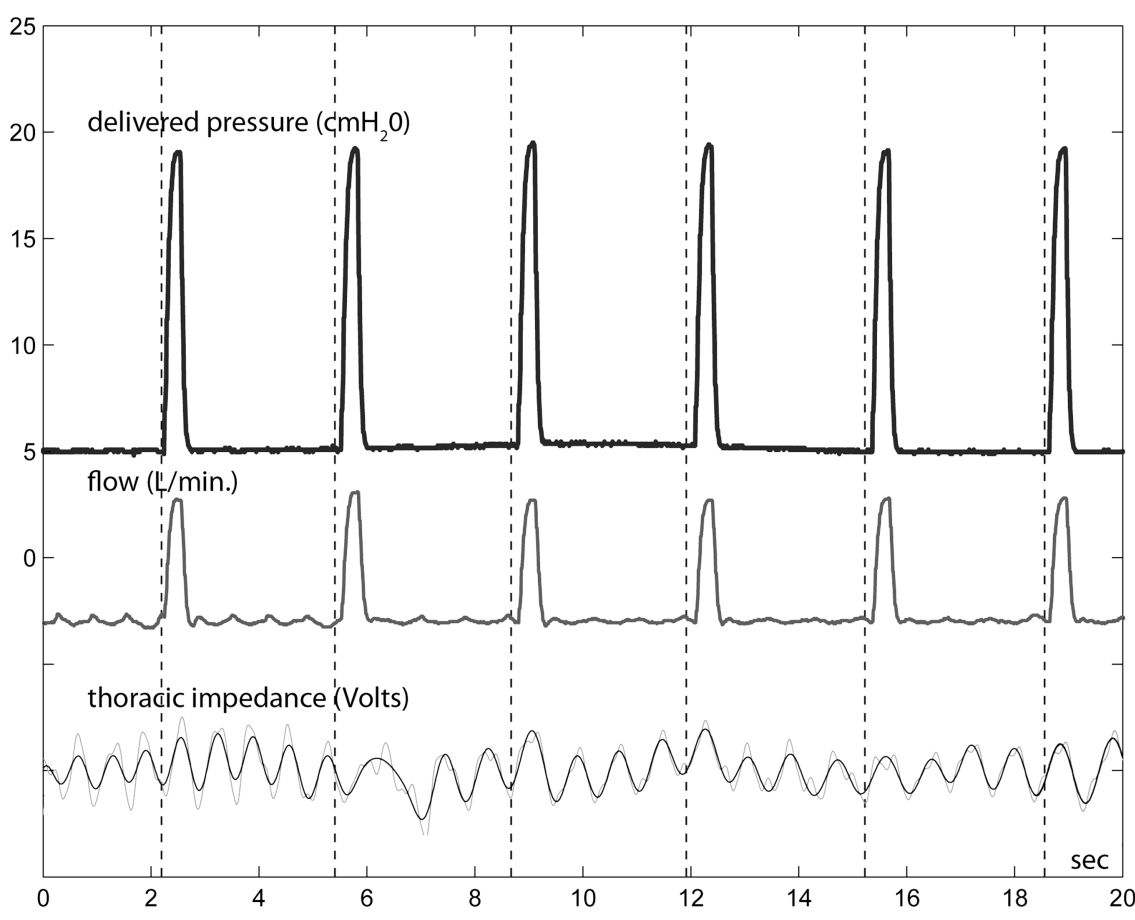

A common criticism of using a flow sensor for NIV is that its reliability can be altered by the continuous flow passing through it, generated by the variable leaks from the infant's nostrils and mouth. To obtain synchronisation, the study ventilator's software elaborates the flow signal detected by the pneumotach. The steady component of flow generated by the continuous variable leaks is quantified and deducted, while the fast variations of flow due to spontaneous breathing are used to trigger the ventilator. The reliability of the system has been tested in a simulated neonatal model, showing that its performance is not affected by the leaks. ${ }^{37}$

A technical limitation possibly affecting our results is that cardiac artefacts may impair the standard impedance pneumography in the detection of central apnoeas. ${ }^{8}$ However, we improved the accuracy of the technique by devising a new filtering strategy, afterwards confirmed by Lee. ${ }^{38}$ Moreover, we were not able to analyse mixed and obstructive apnoeas, due to the concurrent presence of respiratory movements from infants and flow signal from mechanical breaths during flow-SNIPPV and NIPPV. Nevertheless, the distinction between central and mixed/ obstructive spells should not be rigidly maintained, ${ }^{25}$ as obstructive components are commonly involved in apparently purely central apnoeas and vice versa, ${ }^{39} 40$ as indirectly indicated in our study by the observation that in most instances, neither NIPPV nor flow-SNIPPV backup mechanical breaths produced chest wall expansion during central apnoeas.

Finally, statistically significant differences observed in the measured cardiorespiratory parameters according to the nonparametric test were lost on post hoc testing of paired groups, indicating that a larger sample size is needed to correctly interpret these results.

\section{CONCLUSIONS}

Although not conclusive, our study seems to indicate that flow-SNIPPV helps preterm infants in reducing the overall incidence of desaturation and/or bradycardia events and the incidence of central apnoea episodes. Our results, however, need to be validated with a larger number of observations and in infants treated for longer periods.
Acknowledgements The authors thank the parents for letting their infants participate in the study and the nursing staff for its support. The authors also thank Giulio Malerba and Alessandro Veronesi for technical support in collecting data.

Contributors CG had primary responsibility for protocol development, patient screening, enrolment, monitoring data collection for the whole trial, outcome assessment, preliminary and final data analysis and writing the manuscript. She is guarantor. FM participated in the development of the protocol and analytical framework for the study. He performed the analysis and elaboration of recorded signals from monitoring devices involved in the study and contributed to the drafting of the manuscript. VP participated in the development of the protocol, wrote the statistical analysis plan, monitored data collection for the whole trial and contributed to the drafting of the manuscript. CC, ChMa and MC contributed to patient screening and enrolment, supervised and cared for the infants during recordings and monitored data collection for the whole trial. They also contributed to the drafting of the manuscript. PP participated in the development of the protocol providing critical input into study design, helped to perform final data analyses and assisted in drafting the manuscript. CM participated in the development of the protocol, helped to perform final data analyses and assisted in drafting the manuscript. RA supervised the design and execution of the study, performed the final data analyses and contributed to the drafting of the manuscript. All authors read and approved the final manuscript.

Funding This study has been supported by grants from Chiesi Pharmaceuticals and SeSMIT-A.Fa.R (Medical Statistics and Information Technology-Fatebenefratelli Association for Biomedical and Sanitary Research). Chiesi Pharmaceuticals had no role in the study design; in the collection, analysis and interpretation of data; in the writing of the report; and in the decision to submit the paper for publication. Authors CC, CM, and MC received a grant from SeSMIT-A.Fa.R. SeSMIT-A.Fa.R had no role in the study design; in the collection of data; in the writing of the report; and in the decision to submit the paper for publication. VP on behalf of SeSMIT-A. Fa.R performed the statistical analysis of the collected data and she has no competing interests to report.

Competing interests $\mathrm{CM}$ is a consultant to Ginevri Medical Technologies. Ginevri Medical Technologies has not contributed any financial support for this paper or had any part in the authorship.

Patient consent Parental/guardian consent obtained.

Ethics approval The Ethics Committee of "San Giovanni Calibita" Fatebenefratelli Hospital.

Provenance and peer review Not commissioned; externally peer reviewed

\section{REFERENCES}

1 Back SA, Han BH, Luo NL, et al. Selective vulnerability of late oligodendrocyte progenitors to hypoxia-ischemia. J Neurosci 2002;22:455-63. 
2 Janvier A, Khairy M, Kokkotis A, et al. Apnea is associated with neurodevelopmental impairment in very low birth weight infants. J Perinatol 2004; $24: 763-8$.

3 Pillekamp F, Hermann C, Keller T, et al. Factors influencing apnea and bradycardia of prematurity-implications for neurodevelopment. Neonatology 2007;91:155-61.

4 Martin RJ, Wang K, Köroğlu 0, et al. Intermittent hypoxic episodes in preterm infants: do they matter? Neonatology 2011;100:303-10.

5 Ryan CA, Finer NN, Peters KL. Nasal intermittent positive pressure ventilation offers no advantages over nasal continuous positive airway pressure in apnea of prematurity. AJDC 1989;143:1196-8.

6 Lin CH, Wang ST, Lin YJ, et al. Efficacy of nasal intermittent positive pressure ventilation in treating apnea of prematurity. Pediatr Pulmonol 1998;26:349-53.

7 Lemyre B, Davis PG, De Paoli AG. Nasal intermittent positive pressure ventilation (NIPPV) versus nasal continuous positive airway pressure (NCPAP) for apnea of prematurity. Cochrane Database Syst Rev 2002;1:CD002272.

8 Upton CJ, Milner AD, Stokes GM. Combined impedance and inductance for the detection of apnoea of prematurity. Early Hum Dev 1990;24:55-63.

9 Pantalitschka T, Sievers J, Urschitz MS, et al. Randomised crossover trial of four nasal respiratory support systems for apnoea of prematurity in very low birth weight infants. Arch Dis Child Fetal Neonatal Ed 2009;94:F245-8.

10 Speidel BD, Dunn PM. Effect of continuous positive airway pressure on breathing pattern of infants with respiratory-distress syndrome. Lancet 1975;1:302-4.

11 McNamara F, Harris MA, Sullivan CE. Effects of nasal continuous positive airway pressure on apnoea index and sleep in infants. J Pediatr Child Health 1995;31:88-94.

12 Edwards BA, Sands SA, Feeney C, et al. Continuous positive airway pressure reduces loop gain and resolves periodic central apneas in the lamb. Respir Physiol Neurobiol 2009;168:239-49.

13 Hausdorf G, Hellwege HH. Influence of positive end-expiratory pressure on cardiac performance in premature infants: a Doppler-echocardiographic study. Crit Care Med 1987;15:661-4.

14 Trang TT, Tibballs J, Mercier JC, et al. Optimization of oxygen transport in mechanically ventilated newborns using oximetry and pulsed Doppler-derived cardiac output. Crit Care Med 1988:16:1094-7.

15 Hsu HS, Chen W, Wang NK. Effect of continuous positive airway pressure on cardiac output in neonates. Zhonghua Min Guo Xiao Er Ke Yi Xue Hui Za Zhi 1996;37:353-6.

16 Morley CJ, Davis PG, Doyle LW, et al. Nasal CPAP or intubation at birth for very preterm infants. N Engl J Med 2008;358:700-8.

17 Moretti C, Gizzi C, Papoff P, et al. Comparing the effects of nasal synchronized intermittent positive pressure ventilation (nSIPPV) and nasal continuous positive airway pressure (nCPAP) after extubation in very low birth weight infants. Early Hum Dev 1999;56:167-77

18 Owen LS, Morley CJ, Dawson JA, et al. Effects of non-synchronised nasal intermittent positive pressure ventilation on spontaneous breathing in preterm infants. Arch Dis Child Fetal Neonatal Ed 2011;96:F422-8.

19 Moreau-Bussiére F, Samson N, St-Hilaire M, et al. Laryngeal response to nasal ventilation in non sedated newborn lambs. J Appl Physiol 2007;102:2149-57.

20 Praud JP, Samson N, Moreau-Bussière F. Laryngeal function and nasal ventilatory support in the neonatal period. Paediatr Respir Rev 2006;7:S180-2.

21 Hutchison AA, Bignall S. Non-invasive positive pressure ventilation in the preterm neonate: reducing endotrauma and the incidence of bronchopulmonary dysplasia. Arch Dis Child Fetal Neonatal Ed 2008:93:F64-8.

22 Greenough A, Morley C, Davis J. Interaction of spontaneous respiration with artificial ventilation in preterm babies. J Pediatr 1983;103:769-73.
23 Perlmann JM, McMenamin JB, Volpe JJ. Fluctuating cerebral blood-flow velocity in respiratory distress syndrome. Relation to the development of intraventricular haemorrhage. N Engl J Med 1983;309:204-9.

24 Greenough A, Dimitriou G, Prendergast M, et al. Synchronized mechanical ventilation for respiratory support in newborn infants. Cochrane Database Syst Rev 2008;1:CD000456.

25 Poets FC. Apnea of prematurity: What can observational studies tell us about pathophysiology? Sleep Med 2010;11:701-7.

26 Kiciman NM, Andreasson B, Bernstein G, et al. Thoracoabdominal motion in newborns during ventilation delivered by endotracheal tube or nasal prongs. Pediatr Pulmonol 1998;25:175-81.

27 Aghai ZH, Saslow JG, Nakhla T, et al. Synchronized nasal intermittent positive pressure ventilation (SNIPPV) decreases work of breathing (WOB) in premature infants with respiratory distress syndrome (RDS) compared to nasal continuous positive airway pressure (NCPAP). Pediatr Pulmonol 2006;41:875-81.

28 Chang HY, Claure N, D'Ugard C, et al. Effects of synchronization during nasal ventilation in clinically stable preterm infants. Pediatr Res 2011;69:84-9.

29 Barrington KJ, Bull D, Finer NN. Randomized trial of nasal synchronized intermittent mandatory ventilation compared with continuous positive airway pressure after extubation of very low birth weight infants. Pediatrics 2001;107:638-41.

30 Khalaf MN, Brodsky N, Hurley J, et al. A prospective randomized, controlled trial comparing synchronized nasal intermittent positive pressure ventilation versus nasal continuous positive airway pressure as modes of extubation. Pediatrics 2001:108:13-17.

31 Friedlich $\mathrm{P}$, Lecart $\mathrm{C}$, Posen $\mathrm{R}$, et al. A randomized trial of nasopharygeal-synchronized intermittent mandatory ventilation versus nasopharyngeal continuous positive airway pressure in very low birth weight infants after extubation. J Perinatol 1999:19:413-18

32 Santin R, Brodsky N, Bhandari V. A prospective observational pilot study of synchronized nasal intermittent positive pressure ventilation (SNIPPV) as a primary mode of ventilation in infants $>$ or $=28$ weeks with respiratory distress syndrome (RDS). J Perinatol 2004;24:487-93.

33 Moretti C, Giannini L, Fassi C, et al. Nasal flow-synchronized intermittent positive pressure ventilation to facilitate weaning in very low-birth weight infants: Unmasked randomized controlled trial. Pediatr Int 2008;50:85-91.

34 Gizzi C, Papoff P, Giordano I, et al. Flow-synchronized nasal intermittent positive pressure ventilation for infants $<32$ weeks' gestation with respiratory distress syndrome. Crit Care Res Pract 2012;2012:301818.

35 Stein $\mathrm{H}$, Fireston K. Application of neurally adjusted ventilatory assist in neonates. Semin Fetal Neonatal Med 2014;19:60-9.

36 Eichenwald EC, Howell RG III, Kosch PC, et al. Developmental changes in sequential activation of laryngeal abductor muscle and diaphragm in infants. J App/ Physiol 1992;73:1425-31.

37 Moretti C, Papoff P, Gizzi C, et al. Flow-synchronized nasal intermittent positive pressure ventilation in the preterm infant: development of a project. JPNIM 2013;2: e020211.

38 Lee $H$, Rusin CG, Lake DE, et al. A new algorithm for detecting central apnea in meonates. Physiol Meas 2012;33:1-17.

39 Lemke RP, Idiong N, Al-Saedi S, et al. Evidence of a critical period of airway instability during central apneas in preterm infants. Am J Respir Crit Care Med 1998; 157:470-4

40 Al-Sufayan F, Bamehrez M, Kwiatkowski K, et al. The effects of airway closure in central apneas and obstructed respiratory efforts in mixed apneas in preterm infants. Pediatr Pulmonol 2009;44:253-9. 